Check for updates

FAST TRACK
OPEN ACCESS

\section{Effectiveness of the CoronaVac vaccine in older adults during a gamma variant associated epidemic of covid-19 in Brazil: test negative case-control study}

\author{
Otavio T Ranzani,, ${ }^{1,2}$ Matt D T Hitchings, ${ }^{3}$ Murilo Dorion, ${ }^{4}$ Tatiana Lang D’Agostini, ${ }^{5}$ \\ Regiane Cardoso de Paula, ${ }^{5}$ Olivia Ferreira Pereira de Paula, ${ }^{5}$ Edlaine Faria de Moura Villela, ${ }^{6}$ \\ Mario Sergio Scaramuzzini Torres, ${ }^{6}$ Silvano Barbosa de Oliveira, ${ }^{7,8}$ Wade Schulz, ${ }^{9}$ \\ Maria Almiron, ${ }^{7}$ Rodrigo Said, ${ }^{7}$ Roberto Dias de Oliveira, ${ }^{10}$ Patricia Vieira da Silva, ${ }^{11}$ \\ Wildo Navegantes de Araújo, ${ }^{7,8,12}$ Jean Carlo Gorinchteyn, ${ }^{13}$ Jason R Andrews, ${ }^{14}$ \\ Derek A T Cummings, ${ }^{15,16}$ Albert I Ko, ${ }^{4,17}$ Julio Croda ${ }^{4,11,18}$
} For numbered affiliations see
end of the article

Correspondence to: J Croda julio.croda@fiocruz.br

(or @juliocroda on Twitter; Additional material is published online only. To view please visit the journal online.

Cite this as: BMJ 2021;374:n2015 http://dx.doi.org/10.1136/bmj.n2015 Accepted: 12 August 2021 ORCID 0000-0002-6665-6825)

\begin{abstract}
OBJECTIVE

To estimate the effectiveness of the inactivated whole virus vaccine, CoronaVac (Sinovac Biotech), against symptomatic covid-19 in the elderly population of São Paulo state, Brazil during widespread circulation of the gamma variant.

DESIGN

Test negative case-control study.

SETTING

Community testing for covid-19 in São Paulo state, Brazil.
\end{abstract}

\section{PARTICIPANTS}

43774 adults aged $\geq 70$ years who were residents of São Paulo state and underwent reverse transcription polymerase chain reaction (RT-PCR) testing for SARSCoV-2 from 17 January to 29 April 2021. 26433 cases with symptomatic covid-19 and 17622 test negative controls with covid-19 symptoms were formed into 13283 matched sets, one case with to up to five controls, according to age, sex, self-reported race, municipality of residence, previous covid-19 status, and date of RT-PCR test ( \pm 3 days).

INTERVENTION

Vaccination with a two dose regimen of CoronaVac.

\section{WHAT IS ALREADY KNOWN ON THIS TOPIC}

Estimates of effectiveness of the inactivated whole virus vaccine, CoronaVac

(Sinovac Biotech), against symptomatic covid-19 in randomised controlled trials have varied ( $51 \%$ to $84 \%$ )

Current evidence is limited on whether CoronaVac is effective against covid-19 associated severe disease or death, or in the setting of extensive circulation of the gamma variant

More evidence is needed for the real world effectiveness of CoronaVac and other inactivated vaccines among elderly people, a population that has been underrepresented in trials of these vaccines

\section{WHAT THIS STUDY ADDS}

A two dose regimen of CoronaVac was associated with $47 \%$ protection against symptomatic covid-19, 56\% against hospital admissions, and $61 \%$ against deaths among adults aged $\geq 70$ years in the setting of widespread transmission of the gamma variant

Protection is low until $\geq 14$ days after the second dose of CoronaVac

The effectiveness of CoronaVac was observed to decline with increasing age in the elderly population

\section{MAIN OUTCOME MEASURES}

RT-PCR confirmed symptomatic covid-19 and associated hospital admissions and deaths.

\section{RESULTS}

Adjusted vaccine effectiveness against symptomatic covid-19 was $24.7 \%$ (95\% confidence interval $14.7 \%$ to $33.4 \%$ ) at $0-13$ days and $46.8 \%$ (38.7\% to $53.8 \%$ ) at $\geq 14$ days after the second dose. Adjusted vaccine effectiveness against hospital admissions was $55.5 \%$ ( $46.5 \%$ to $62.9 \%$ ) and against deaths was $61.2 \%$ ( $48.9 \%$ to $70.5 \%$ ) at $\geq 14$ days after the second dose. Vaccine effectiveness $\geq 14$ days after the second dose was highest for the youngest age group (70-74 years) $-59.0 \%$ (43.7\% to $70.2 \%)$ against symptomatic disease, $77.6 \%$ (62.5\% to $86.7 \%$ ) against hospital admissions, and $83.9 \%$ (59.2\% to $93.7 \%$ ) against deaths-and declined with increasing age.

\section{CONCLUSIONS}

Vaccination with CoronaVac was associated with a reduction in symptomatic covid-19, hospital admissions, and deaths in adults aged $\geq 70$ years in a setting with extensive transmission of the gamma variant. Vaccine protection was, however, low until completion of the two dose regimen, and vaccine effectiveness was observe to decline with increasing age among this elderly population.

\section{Introduction}

As of early July 2021 the covid-19 pandemic has been responsible for 3.9 million deaths worldwide, ${ }^{1}$ with a disproportionately high mortality and morbidity among elderly people. ${ }^{2}$ A key question is whether the authorised covid-19 vaccines are effective in elderly people, who might have impaired immune responses ${ }^{34}$ and are underrepresented in randomised controlled trials. ${ }^{5-7}$ mRNA and adenovirus vector based vaccines have been shown to be effective against covid-19 in elderly people, ${ }^{89}$ but evidence on the effectiveness of inactivated vaccines in this population is limited. ${ }^{7}{ }^{10-12}$

CoronaVac (Sinovac Biotech), an inactivated whole virus vaccine, has been approved by 32 countries and jurisdictions ${ }^{10}$ and been implemented as part of mass vaccination campaigns in low and middle income countries, many of which are experiencing covid-19 epidemics as a result of the emergence of SARS-CoV-2 variants of concern. Estimates from randomised controlled trials of vaccine efficacy 
against symptomatic covid-19 of a two dose CoronaVac regimen in healthcare workers and the general population have varied (51\% to $84 \%) .{ }^{5710}$ The World Health Organization's Emergency Use Listing (EUL) procedure approved the use of CoronaVac in early June 2021 but identified an evidence gap for the effectiveness of this vaccine in adults aged $\geq 60$ years. ${ }^{11}$ The WHO EUL cited an observational study in Chile, ${ }^{1012}$ which found that the adjusted effectiveness of CoronaVac among adults aged $\geq 60$ years at 14 days or more after the second dose was $66.6 \%$. During the study period, the gamma variant of concern was detected in $28.6 \%$ of SARS-CoV-2 genomes in Chile. ${ }^{12}$ Furthermore, randomised controlled trials and observational studies have not investigated whether CoronaVac provides important protection after the first dose or in the setting of widespread transmission of variants of concern. ${ }^{51011}$

Brazil has experienced one of the world's highest covid-19 burdens during the pandemic, with more than 18 million people affected and 526000 deaths reported as of early July 2021. ${ }^{113}$ Variants of concern, and in particular the gamma variant, have played an important role in the recent epidemic wave in Brazil, which began in early $2021 .^{14-16}$ The gamma variant, which was first detected in Manaus, shows increased transmissibility, ${ }^{16}$ has accrued mutations associated with decreased in vitro seroneutralisation, ${ }^{17-19}$ has a possible association with increased disease severity, ${ }^{2021}$ and, at present, accounts for most of the SARS-CoV-2 isolates genotyped in Brazil from 1 January $2021 .^{14} 22$ In the setting of a large epidemic associated with the gamma variant in São Paulo, the most populous state in Brazil, we conducted a matched, test negative ${ }^{23}$ case-control study to evaluate the real world effectiveness of CoronaVac against symptomatic covid-19 and severe clinical outcomes in people aged $\geq 70$ years.

\section{Methods}

\section{Study setting}

The State of São Paulo $\left(23^{\circ} 3^{\prime} \mathrm{S}, 46^{\circ} 4^{\prime} \mathrm{W}\right)$ has 645 municipalities and 46 million inhabitants, 3.23 million of whom are aged $\geq 70$ years. $^{24}$ The state experienced three successive waves of covid-19, during which 2997282 cases (cumulative incidence rate: 6475 per 100000 population) and 100649 deaths (cumulative mortality: 217 per 100000 population) have been reported as of 9 May 2021 (fig 1, supplementary figure 1). ${ }^{25}$ The state secretary of health of Sao Paulo initiated a covid-19 vaccination campaign for the general population on 17 January 2021 according to an age based prioritisation strategy (fig 1) and is administering a two dose regimen of CoronaVac with a two to four week interval between doses, and a two dose regimen of ChAdOx1 nCoV-19 (Oxford-AstraZeneca), with a 12 week interval. ${ }^{26}$ As of 29 April 2021, 8.63 million doses (5.16 million first doses and 3.47 million second doses) of CoronaVac had been administered and 2.06 million doses (1.99 million first doses and 0.07 million second doses) of ChAdOx1 nCoV-19.

\section{Study design}

We conducted a matched test negative case-control study to estimate the effectiveness of CoronaVac in reducing the odds of reverse transcription polymerase chain reaction (RT-PCR) confirmed symptomatic covid-19 in adults aged $\geq 70$ years from São Paulo state from 17 January 2021 (the start of covid-19 vaccination) to 29 April 2021. Test negative design studies have provided estimates of vaccine effectiveness in concordance with those obtained from randomised controlled trials, ${ }^{27} 28$ and such studies have been used extensively to evaluate vaccines against respiratory infections, ${ }^{29}$ including covid-19. ${ }^{82330}$ We chose the test negative design because of the feasibility of accessing information on people who were tested for SARS-CoV-2 through the São Paulo state surveillance systems and because of the opportunity to control for potential biases, such as healthcare seeking behaviour and access to testing. ${ }^{23}$ The study population was adults aged $\geq 70$ years who had a residential address in São Paulo state, underwent SARS-CoV-2 RT-PCR testing during the study period, and had complete and consistent information between data sources on age, sex, residence, and on vaccination and testing status and dates. We matched test negative controls with covid-19 symptoms to covid-19 cases by date of testing ( \pm 3 days) to address potential sources of bias that might vary during the course of an epidemic, as well as by participant characteristics of age, sex, selfreported race, municipality of residence, and previous covid-19 status.

In the protocol, we prespecified power thresholds for conducting analyses on the effectiveness of CoronaVac and ChAdOx1 nCoV-19. These thresholds were achieved for CoronaVac but not for ChAdOx1 nCoV-19 because of lower rates of ChAdOx1 nCov19 administered in the population during the study period. We therefore restricted the evaluation of vaccine effectiveness to CoronaVac.

\section{Amendment of protocol}

The study design and statistical analysis plan were specified in advance of extracting information from data sources and are described in a publicly available protocol (https://github.com/juliocroda/ VebraCOVID-19) and the supplementary file. We made two major changes to the original protocol: we added the analysis for hospital admissions and deaths inside the framework of a test negative design before submission to peer review (post hoc analysis), and after submission, as suggested by peer reviewers, we changed the matching procedure of the main analysis from one case matched to one control without replacement, to one case matched with up to five controls with replacement of controls between cases (unbalanced design); and we added two other sensitivity analyses for the matching procedure.

\section{Data sources}

We obtained individual level information on personal characteristics, comorbidities, SARS-CoV-2 testing, 

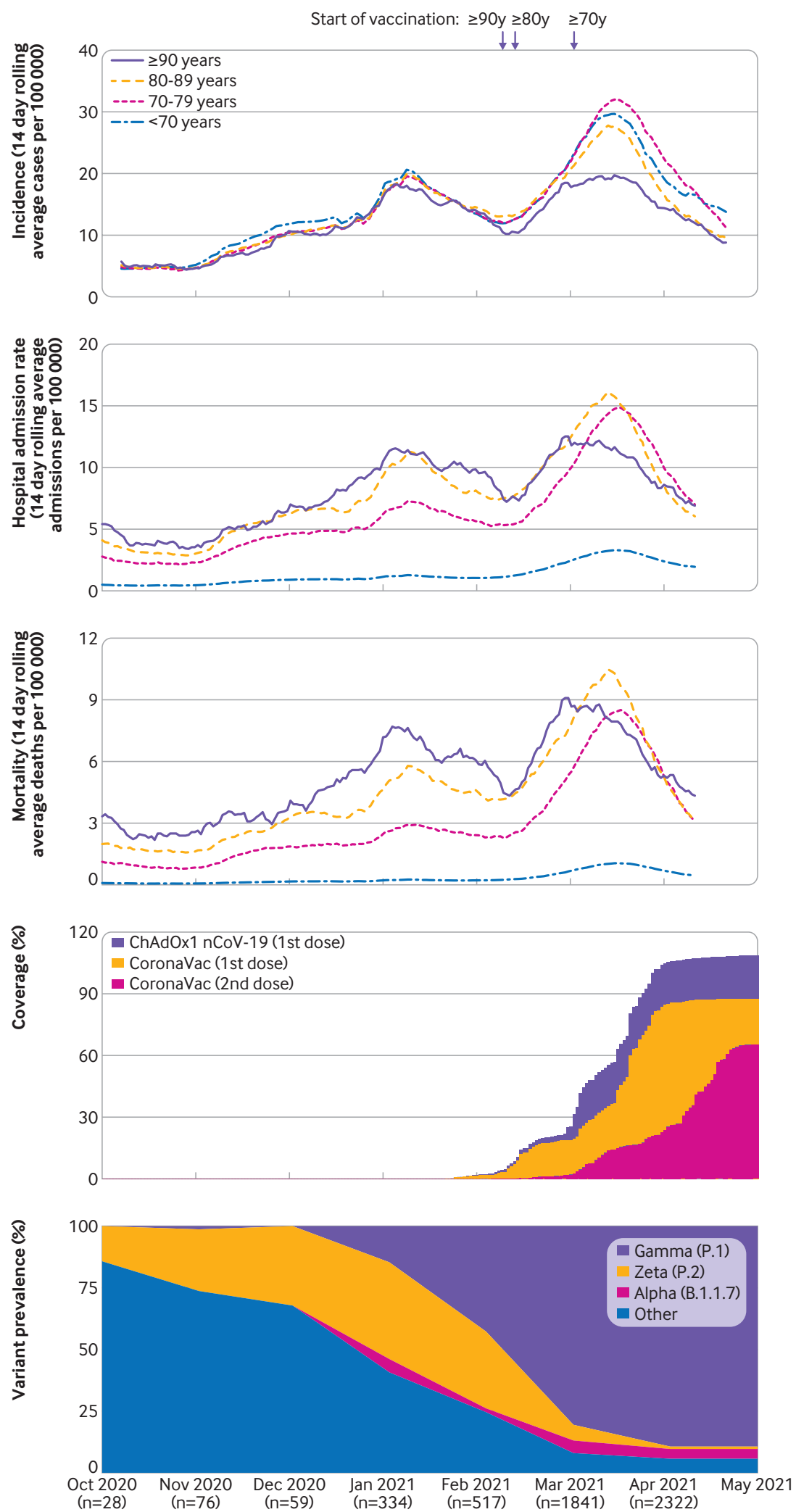

Fig 1 I Incidence of reported covid-19, vaccination coverage, and prevalence of SARS-CoV-2 variants of concern from 1 October 2020 to 29 April 2021 in São Paulo state, Brazil. Panels A-C show the 14 day rolling average of daily age group specific incidence of reported covid-19 cases, hospital admission rate, and mortality (events per 100000 population). Panel $D$ shows daily cumulative vaccination coverage in people aged $\geq 70$ years. Population estimates for age groups were obtained from national projections for $2020 .{ }^{24}$ Panel E shows the monthly prevalence of SARSCoV-2 variants among genotyped isolates in the GISAID (global initiative on sharing avian influenza data) database (extraction on 20 June 2021). ${ }^{22}$ Vertical bars show dates that adults aged $\geq 90,80-89$, and $70-79$ years in the general population became eligible for vaccination 
and covid-19 vaccination during the study period by extracting information on 6 May 2021 from the state secretary of health of Sao Paulo laboratory testing registry (GAL), the national surveillance databases for covid-19-like illnesses (e-SUS) and severe acute respiratory illness (SIVEP-Gripe), and the state secretary of health of Sao Paulo vaccination registry (Vacina Já). All people living in Brazil are eligible for testing and have access to the public health system. RT-PCR tests are performed by trained healthcare professionals following standard protocols. Notification of people with suspected covid-19, SARSCoV-2 test results, and suspected deaths with covid-19 is compulsory in Brazil. Supplementary table 1 provides additional information on data sources. The information technology bureau of the São Paulo state government linked individual level records from the four databases using CPF (Cadastro de Pessoas Físicas) numbers (Brazilian citizens' unique identifier code) and provided anonymised datasets. The genotyping of all isolated SARS-CoV-2 in São Paulo state was not possible and these data are not available in the surveillance systems used in this study. We retrieved information on SARS-CoV-2 variants from genotyped isolates from São Paulo state deposited in the global initiative on sharing avian influenza data (GISAID) database. $^{22}$

\section{Selection of cases and matched controls}

We selected cases from the study population who had covid-19 symptoms, defined as a covid-19-like illness, a positive SARS-CoV-2 RT-PCR test result from a respiratory sample that was collected within 10 days after the onset of symptoms, and did not have a positive RT-PCR test result in the preceding 90 days. We selected controls from the study population who had a covid-19-like illness, a negative SARS-CoV-2 RT-PCR test result from a respiratory sample that was collected within 10 days after the onset of symptoms, ${ }^{23}$ and no positive RT-PCR test result in the previous 90 days during the study period, or in the subsequent 14 days. Cases and controls were excluded if they received the ChAdOx1 nCoV-19 vaccine before sample collection for RT-PCR testing. We defined covid-19-like illness as the presence of one or more reported covid-19 related symptoms. ${ }^{31}$

One case was matched with to up to five test negative controls according to RT-PCR sample collection date ( \pm 3 days), age category (five year age bands, eg, 7074, 75-79 years), sex, municipality of residence, selfreported race (defined as brown, black, yellow, white, or indigenous), ${ }^{32}$ and previous symptomatic events that were reported to the surveillance systems ${ }^{31}$ between 1 February 2020 and 16 January 2021, as a proxy for previous SARS-CoV-2 infection. Matching factors were chosen from variables that were associated with vaccination coverage or timing, and with risk of SARSCoV-2 infection or healthcare access (see protocol in supplementary file). ${ }^{23}$ After identification of each case, we randomly chose up to five controls in an unbalanced design from the set of all eligible matching controls, allowing for replacement of controls between cases (main analysis). We conducted three sensitivity analyses, varying two features of the matching while keeping the same matching factors. In the first analysis we matched one case to one random control without replacement of controls (original analysis in the protocol); in the second analysis we matched one case to one random control, allowing for replacement of controls between cases; and in the third analysis we matched one case to two random controls, allowing for replacement of controls between cases.

\section{Statistical analysis}

We estimated the effectiveness of CoronaVac against symptomatic covid-19 in the 0-13 days and $\geq 14$ days after the second dose and $\geq 14$ days after the first dose. Furthermore, we estimated the effectiveness of a single dose 0-13 days after the first dose, when the vaccine has shown no or limited effectiveness. ${ }^{5} 3334$ An association during this period might serve as an indicator of unmeasured confounding in the effectiveness estimate. ${ }^{3536}$ We also expanded our bias indicator by evaluating the 0-13 days after the first dose as 0-6 days and 7-13 days. ${ }^{36}$ The reference group for vaccination status was those who had not received a first vaccine dose before the date of sample collection.

Conditional logistic regression was used to estimate the odds ratio of vaccination among cases and controls: 1-odds ratio provided an estimate of vaccine effectiveness under the assumptions of a test negative design. ${ }^{37}$ We included age and covid-19 associated comorbidities (cardiovascular, renal, neurological, haematological, and hepatic, diabetes, chronic respiratory disorder, obesity, or immunosuppression) as covariates in the model. Because age is a strong determinant of covid-19 outcomes, we adjusted for age after matching by age bands to control for potential residual confounding. ${ }^{38}$ Non-linearity for age was evaluated using restricted cubic splines and we chose the most parsimonious model comparing nested models with a likelihood ratio test. To evaluate potential residual confounding by time varying factors that might not be dealt with by the matching criteria, we also conducted a post hoc sensitivity analysis that incorporated the calendar date of RT-PCR sample collection in the model.

In a post hoc analysis we estimated vaccine effectiveness against covid-19 associated hospital admission and death. To account for the competing event of dying before being admitted to hospital, we estimated vaccine effectiveness for the composite outcome of hospital admission or death, or both in a sensitivity analysis. In these separate analyses, we selected matched pairs in which the case had the secondary outcome of interest. ${ }^{39}{ }^{40}$ We fit the same conditional logistic regression model as for the primary outcome.

We conducted a prespecified analysis of vaccine effectiveness among age subgroups for the primary and secondary outcomes but could not perform analyses stratified by previous covid-19 documented infection 
because of small numbers. The age subgroups were prespecified and followed the five year age categories used for matching, with age groups older than 80 collapsed into a single group. Additional post hoc analyses were performed of vaccine effectiveness for the primary outcome for subgroups stratified by sex, number of chronic comorbidities (none versus at least one), the two most common chronic comorbidities (cardiovascular disease and diabetes), and region of residence (Grande São Paulo health region versus others). Interaction terms were incorporated into the model to evaluate the association of each subgroup of interest with vaccine effectiveness $\geq 14$ days after the second dose.

\section{Power calculation}

Our protocol specified that we would conduct proposed analyses after achieving $\geq 80 \%$ power to identify a vaccine effectiveness of $40 \%$ against symptomatic covid-19 for $\geq 14$ days after the second dose of CoronaVac compared with not receiving a vaccine dose. The power was simulated fitting conditional logistic regressions on 1000 simulated datasets. After extracting information from the surveillance databases on 6 May 2021 and generating matched case-control pairs, we determined that the power of the study was 99.9\% and proceeded to conduct the prespecified analyses. We did not perform an analysis for ChAdOx1 nCoV-19 because the simulated power was $31 \%$ to identify a vaccine effectiveness of $40 \%$ for $\geq 28$ days after the first dose of ChAdOx1 nCoV-19 compared with not receiving a vaccine dose.

All analyses were done in $\mathrm{R}$, version 4.0.2.

\section{Patient and public involvement}

Because this study used routine surveillance data sources and there was no direct funding, no members of the public or patients were directly involved. Nevertheless, we did speak to patients about the study and the outcomes to be evaluated, and we asked a member of the public to read our manuscript and provide inputs for its interpretation. No members of the public or patients were involved in writing up the results.

\section{Results}

São Paulo state experienced three covid-19 epidemic waves, with a peak incidence in July 2020 for the first wave and in January 2021 for the second wave (supplementary figure 1) and March 2021 for the third wave (fig 1). The second wave was preceded in November 2020 by an increase in the prevalence of the zeta variant among genotyped isolates from São Paulo state deposited into the GISAID database (fig 1). The third wave was preceded in January 2021 by an increase in the prevalence of the gamma variant among genotyped isolates (fig 1). The gamma variant replaced other SARS-CoV-2 variants ${ }^{22}$ and accounted for $78.4 \%$ (3834/4887) of the genotyped isolates that were reported in GISAID during the study period and $85.5 \%$ (3584/4192) of genotyped isolates that were reported between 1 March and 29 April 2021, when the majority of discordant case-control sets were identified (supplementary figure 2). The vaccination campaign, initiated on 17 January 2021, achieved an estimated coverage of roughly $85 \%$ for the first CoronaVac dose (2.82 million) and $65 \%$ for the second dose $(2.10$ million) among adults aged $\geq 70$ years by 29 April 2021 (fig 1). After initiation of the vaccination campaign and during the third epidemic wave, the incidence of covid-19 increased and peaked in late March in all age groups except for those aged $\geq 90$ years (fig 1 ).

\section{Study population}

From 17 January 2021 to 29 April 2021, the rate of RT-PCR testing for SARS-CoV-2 in those age $\geq 70$ years in São Paulo state was 25 per 1000 persons. Among 43774 adults eligible for study inclusion (fig 2), 22177 (50.7\%) who provided 55519 RT-PCR test results were included in matched case and control sets as follows: 3881 pairs matched 1:1, 1963 pairs matched 1:2, 1044 pairs matched 1:3, 678 pairs matched 1:4, and 5717 pairs matched 1:5. Overall, 6223 participants contributed more than one time as controls and 18 participants contributed as both control and case. Table 1 shows the characteristics of eligible participants with positive and negative RT-PCR test results, and selected cases and matched controls. Matched characteristics appear unbalanced because of the variable matching procedure. A higher proportion of cases than controls had reported comorbidities. Most of the discordant sets, based on vaccination status, were selected after 14 March 2021 (supplementary figure 2). For cases and controls who completed the two dose vaccine regimen, the intervals between doses were similar (mean $30 v 25$ days). Likewise, the intervals between vaccine doses and RT-PCR testing were similarly distributed for cases and controls (table 1 and supplementary figure 3). Supplementary table 2 shows the distribution of matched sets according to the vaccination status of cases and controls at the time of RT-PCR testing. Supplementary tables 3 and 4 show the characteristics of the matched case and control sets selected for the analysis of secondary outcomes of hospital admission $(n=30308)$ and death $(n=14624)$.

Vaccine effectiveness against symptomatic covid-19 The adjusted effectiveness against symptomatic covid-19 was $24.7 \%$ (95\% confidence interval $14.7 \%$ to $33.4 \%$ ) at 0-13 days and $46.8 \%$ (38.7\% to $53.8 \%$ ) at $\geq 14$ days after the second dose (table 2). No statistically significant change was identified in the odds of covid-19 in the 0-13 days after the first dose, which serves as a potential bias indicator. The bias indicator was similar 0-6 days and 7-13 days after the first dose (supplementary table 5). In the sensitivity analysis including calendar date of testing as a covariate, vaccine effectiveness after the second dose was $25.1 \%$ (15.2\% to $33.8 \%$ ) at $0-13$ days and $47.1 \%$ (39.1\% to $54.1 \%$ ) at $\geq 14$ days.

Vaccine effectiveness against symptomatic covid-19 was observed to decline with increasing age $\geq 14$ 


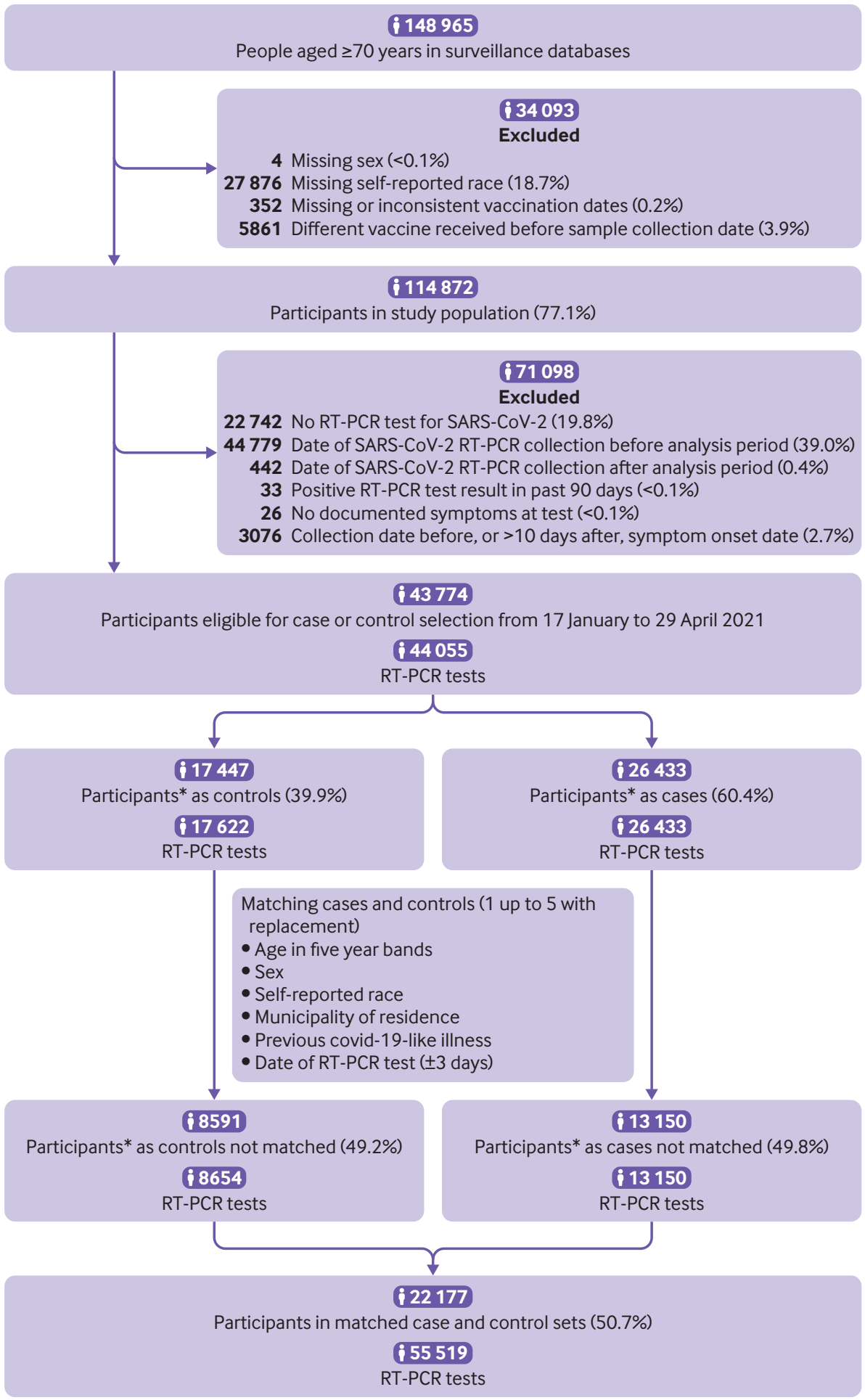

Fig 2 | Flowchart of study population from surveillance databases, and selection of matched cases and controls. * Some participants contributed as controls and cases, and matching allowed for replacement of controls between cases. $\mathrm{RT}-\mathrm{PCR}=$ reverse transcription polymerase chain reaction

days after the second dose and was 59.0\% (43.7\% to $70.2 \%$ ) among those aged $70-74$ years, $56.2 \%$ (43.0\% to 66.3\%) among those aged $75-79$ years, and $32.7 \%(17.0 \%$ to $45.5 \%)$ among those aged $\geq 80$ years $(\mathrm{P}=0.007$ for interaction; figure 3 , supplementary table 6). Vaccine effectiveness against symptomatic covid-19 did not differ among subgroups defined by sex, presence of comorbidities, reported cardiovascular disease, or regions of residence. Participants with reported diabetes, however, had lower protection than those without reported diabetes (vaccine effectiveness $32.6 \% v 50.5 \%, \mathrm{P}=0.008$ for interaction) $\geq 14$ days after the second dose (supplementary table 7 and supplementary figure 4) 


\begin{tabular}{|c|c|c|c|c|}
\hline \multirow[b]{2}{*}{ Characteristics } & \multicolumn{2}{|c|}{ Eligible cases and controls } & \multicolumn{2}{|l|}{ Matched sets } \\
\hline & $\begin{array}{l}\text { Test negative } \\
(\mathrm{n}=17622)^{\star}\end{array}$ & $\begin{array}{l}\text { Test positive } \\
(n=26433)^{*}\end{array}$ & Controls $(n=42236)^{*}$ & Cases $(n=13283)^{\star}$ \\
\hline Mean (SD) age (years) & $77.53(6.8)$ & $76.71(6.2)$ & $75.69(5.44)$ & $75.90(5.64)$ \\
\hline \multicolumn{5}{|l|}{ Age groups (years): } \\
\hline $70-79$ & $12123(68.8)$ & $19673(74.4)$ & $34134(80.8)$ & $10543(79.4)$ \\
\hline $80-89$ & $4301(24.4)$ & $5437(20.6)$ & $7045(16.7)$ & $2311(17.4)$ \\
\hline$\geq 90$ & $1198(6.8)$ & $1323(5.0)$ & $1057(2.5)$ & $429(3.2)$ \\
\hline Men & 7689 (43.6) & $12431(47.0)$ & $18610(44.1)$ & 5919 (44.6) \\
\hline \multicolumn{5}{|l|}{ Self-reported racet: } \\
\hline White/branca & $13415(76.1)$ & $19796(74.9)$ & $34603(81.9)$ & $10803(81.3)$ \\
\hline Brown/pardo & $3192(18.1)$ & $4983(18.9)$ & $6797(16.1)$ & $2115(15.9)$ \\
\hline Black/preta & $785(4.5)$ & $1258(4.8)$ & $727(1.7)$ & $287(2.2)$ \\
\hline Yellow/amarela & $226(1.3)$ & $390(1.5)$ & $109(0.3)$ & $78(0.6)$ \\
\hline Indigenous/Indígena & $4(0.0)$ & $6(0.0)$ & - & - \\
\hline Residence in Grande São Paul health region & $12381(70.3)$ & $16538(62.6)$ & $14368(34.0)$ & $6113(46.0)$ \\
\hline \multicolumn{5}{|l|}{ Reported No of comorbiditiesł: } \\
\hline 0 & $10027(56.9)$ & $12668(47.9)$ & $23961(56.7)$ & $5886(44.3)$ \\
\hline 1 or 2 & $6984(39.6)$ & $12548(47.5)$ & $16626(39.4)$ & $6713(50.5)$ \\
\hline$\geq 3$ & $611(3.5)$ & $1217(4.6)$ & $1649(3.9)$ & $684(5.1)$ \\
\hline Cardiovascular disease & $5293(30.0)$ & $10079(38.1)$ & $12563(29.7)$ & $5482(41.3)$ \\
\hline Diabetes & $3233(18.3)$ & $6533(24.7)$ & $8269(19.6)$ & 3578 (26.9) \\
\hline \multicolumn{5}{|l|}{ Past exposure to SARS-CoV- $2 \S$} \\
\hline Previous symptomatic events notified to surveillance systems $\mathbb{\|}$ & 685 (3.9) & $354(1.3)$ & $47(0.1)$ & $37(0.3)$ \\
\hline Positive SARS-CoV-2 test result** & $66(0.4)$ & $13(0.0)$ & $1(0.0)$ & $4(0.0)$ \\
\hline $\begin{array}{l}\text { Median (interquartile range) interval between symptoms onset and RT-PCR } \\
\text { testing (days) }\end{array}$ & $3(2-5)$ & $4(2-6)$ & $3(1-5)$ & $4(2-6)$ \\
\hline Hospital admissions & 4524/17484 (25.9) & $12987 / 26221(49.5)$ & $11020 / 41980(26.3)$ & $7043 / 13175(53.5)$ \\
\hline Deaths & $1594 / 16710(9.5)$ & $7054 / 24508(28.8)$ & $4072 / 40134(10.1)$ & $3549 / 12251(29.0)$ \\
\hline $\begin{array}{l}\text { Median (interquartile range) interval between symptoms onset and hospital } \\
\text { admission (days) }\end{array}$ & $3(2-6)$ & $7(4-10)$ & $4(2-7)$ & $7(4-10)$ \\
\hline $\begin{array}{l}\text { Median (interquartile range) interval between symptoms onset and deaths } \\
\text { (days) }\end{array}$ & $8(4-13)$ & $14(9-21)$ & $8(4-16)$ & $15(10-22)$ \\
\hline \multicolumn{5}{|l|}{ Vaccination status: } \\
\hline Not vaccinated & $11986(68.0)$ & $17233(65.2)$ & $27994(66.3)$ & $8989(67.7)$ \\
\hline Single dose, within 0-13 days & $1446(8.2)$ & 2976 (11.3) & $4873(11.5)$ & 1565 (11.8) \\
\hline Single dose, $\geq 14$ days & $1797(10.2)$ & $3312(12.5)$ & $4631(11.0)$ & $1489(11.2)$ \\
\hline Two doses, within 0-13 days & $1041(5.9)$ & $1533(5.8)$ & $2445(5.8)$ & $700(5.3)$ \\
\hline Two doses, $\geq 14$ days & $1352(7.7)$ & $1379(5.2)$ & $2293(5.4)$ & $540(4.1)$ \\
\hline Mean (SD) interval between 1st and 2nd dose (days) & $25(6)$ & $30(12)$ & $25(6)$ & $30(12)$ \\
\hline Mean (SD) interval between 1st dose and RT-PCR testing (days) & $28(19)$ & $23(16)$ & $22(17)$ & $21(16)$ \\
\hline Mean (SD) interval between second dose and RT-PCR testing (days) & $20(15)$ & $17(14)$ & $17(14)$ & $16(14)$ \\
\hline \multicolumn{5}{|c|}{ 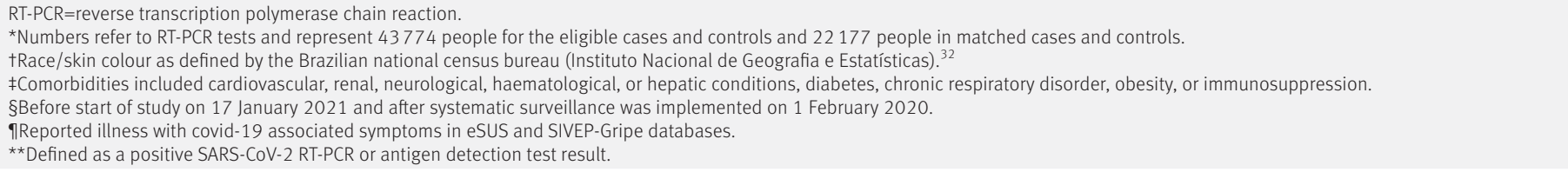 } \\
\hline
\end{tabular}

\section{Vaccine effectiveness against covid-19 associated hospital admissions}

The adjusted effectiveness against hospital admission was $39.1 \%(28.0 \%$ to $48.5 \%)$ at $0-13$ days and $55.5 \%$ $(46.5 \%$ to $62.9 \%$ ) at $\geq 14$ days after the second dose (table 2). No statistically significant reduction was observed in the odds of covid-19 in the periods after one dose, and the bias indicator effectiveness was close to zero (supplementary table 5).

Vaccine effectiveness against hospital admission was observed to decline with increasing age $\geq 14$ days after the second dose and was $77.6 \%(62.5 \%$ to $86.7 \%$ ) among those aged $70-74$ years, $66.6 \%$ (51.8\% to $76.9 \%$ ) among those aged $75-79$ years, and $38.9 \%$ (21.4\% to $52.5 \%$ ) among those aged $\geq 80$ years $(\mathrm{P}<0.001$ for interaction; fig 3 , supplementary table 6).
Vaccine effectiveness against deaths with covid-19 The adjusted effectiveness against deaths with covid-19 was $31.2 \%(17.6 \%$ to $42.5 \%) \geq 14$ days after the first dose, $48.9 \%$ (34.4\% to $60.1 \%$ ) 0-13 days after the second dose, and $61.2 \%(48.9 \%$ to $70.5 \%) \geq 14$ days after the second dose (table 2). The bias indicator was close to zero 0-13 days after the first dose, and 0-6 days and 7-13 days after the first dose (supplementary table 5).

Vaccine effectiveness against deaths was observed to decline with increasing age $\geq 14$ days after the second dose and was $83.9 \%$ (59.2\% to $93.7 \%$ ) among those aged $70-74$ years, $78.0 \%$ (58.8\% to $88.3 \%$ ) among those aged $75-79$ years, and $44.0 \%$ (20.3\% to $60.6 \%)$ among those aged $\geq 80$ years $(\mathrm{P}=0.001$ for interaction; fig 3, supplementary table 6). 


\begin{tabular}{|c|c|c|c|c|c|c|}
\hline & \multicolumn{3}{|l|}{ Unadjusted analysis } & \multicolumn{3}{|l|}{ Adjusted analysis* } \\
\hline & Odds ratio $(95 \% \mathrm{Cl})$ & $\begin{array}{l}\text { Vaccine effectiveness, } \\
\%(95 \% \mathrm{Cl})\end{array}$ & $P$ value & Odds ratio $(95 \% \mathrm{Cl})$ & $\begin{array}{l}\text { Vaccine effectiveness, } \\
\%(95 \% \mathrm{Cl})\end{array}$ & $P$ value \\
\hline \multicolumn{7}{|c|}{ Symptomatic covid-19 $(n=55519)$} \\
\hline \multicolumn{7}{|c|}{ One dose: } \\
\hline 0-13 days $v$ unvaccinated $t$ & $1.02(0.94$ to 1.10$)$ & $-1.7(-10.4$ to 6.2$)$ & 0.68 & 1.01 (0.93 to 1.09$)$ & $-0.8(-9.4$ to 7.2$)$ & 0.86 \\
\hline$\geq 14$ days $v$ unvaccinated $\dagger$ & 0.88 (0.80 to 0.97$)$ & 11.9 (3.1 to 19.9$)$ & 0.01 & $0.88(0.79$ to 0.96$)$ & $12.5(3.7$ to 20.6$)$ & 0.01 \\
\hline \multicolumn{7}{|l|}{ Two doses: } \\
\hline 0-13 days $v$ unvaccinated $t$ & $0.77(0.68$ to 0.87$)$ & $23.5(13.5$ to 32.3$)$ & $<0.001$ & $0.75(0.67$ to 0.85$)$ & $24.7(14.7$ to 33.4$)$ & $<0.001$ \\
\hline$\geq 14$ days $v$ unvaccinated $t$ & $0.54(0.47$ to 0.62$)$ & $45.8(37.7$ to 52.9$)$ & $<0.001$ & $0.53(0.46$ to 0.61$)$ & 46.8 (38.7 to 53.8$)$ & $<0.001$ \\
\hline \multicolumn{7}{|c|}{ Hospital admissions associated with covid-19 $(n=30308)$} \\
\hline \multicolumn{7}{|c|}{ One dose: } \\
\hline 0-13 days $v$ unvaccinated $t$ & $0.98(0.89$ to 1.09$)$ & $1.6(-9.3$ to 11.5$)$ & 0.76 & $0.93(0.84$ to 1.04$)$ & $6.6(-4.3$ to 16.3$)$ & 0.23 \\
\hline$\geq 14$ days $v$ unvaccinated $t$ & $0.87(0.77$ to 0.99$)$ & $12.6(1.3$ to 22.6$)$ & 0.03 & $0.83(0.73$ to 0.94$)$ & $16.9(5.7$ to 26.8$)$ & 0.004 \\
\hline \multicolumn{7}{|l|}{ Two doses: } \\
\hline 0-13 days $v$ unvaccinated $t$ & $0.66(0.56$ to 0.77$)$ & $34.4(23.1$ to 44.1$)$ & $<0.001$ & $0.61(0.52$ to 0.72$)$ & 39.1 (28.0 to 48.5$)$ & $<0.001$ \\
\hline$\geq 14$ days $v$ unvaccinated $t$ & $0.48(0.40$ to 0.57$)$ & 51.9 (42.6 to 59.7$)$ & $<0.001$ & $0.45(0.37$ to 0.54$)$ & $55.5(46.5$ to 62.9$)$ & $<0.001$ \\
\hline \multicolumn{7}{|c|}{ Deaths associated with covid-19 $(n=14624)$} \\
\hline \multicolumn{7}{|c|}{ One dose: } \\
\hline 0-13 days $v$ unvaccinated $t$ & $0.90(0.78$ to 1.04$)$ & $10(-4.2$ to 22.2$)$ & 0.16 & $0.87(0.74$ to 1.02$)$ & $13.1(-1.5$ to 25.6$)$ & 0.08 \\
\hline$\geq 14$ days $v$ unvaccinated $t$ & $0.75(0.63$ to 0.89$)$ & 25.1 (11.2 to 36.9) & 0.001 & $0.69(0.58$ to 0.82$)$ & $31.2(17.6$ to 42.5$)$ & $<0.001$ \\
\hline \multicolumn{7}{|l|}{ Two doses: } \\
\hline 0-13 days $v$ unvaccinated $t$ & $0.56(0.44$ to 0.70$)$ & $44.3(29.6$ to 55.9$)$ & $<0.001$ & $0.51(0.40$ to 0.66$)$ & $48.9(34.4$ to 60.1$)$ & $<0.001$ \\
\hline$\geq 14$ days $v$ unvaccinated $t$ & $0.43(0.33$ to 0.56$)$ & $57.1(44.3$ to 67$)$ & $<0.001$ & $0.39(0.30$ to 0.51$)$ & $61.2(48.9$ to 70.5$)$ & $<0.001$ \\
\hline
\end{tabular}

The adjusted effectiveness for the composite outcome hospital admissions or deaths, or both, was $39.2 \%(28.3 \%$ to $48.4 \%) 0-13$ days after the second dose, and $55.4 \%$ ( $46.5 \%$ to $62.8 \%) \geq 14$ days after the second dose (supplementary table 8 ).

Sensitivity analyses for the matching procedure Overall, 13150 cases (49.7\%) could not be matched with a potential control. Thus, $30.1 \%$ of cases (7950 case-control pairs) could be matched in the first sensitivity analysis (1:1 without replacement), 50.3\% of cases (13283 case-control pairs) in the second sensitivity analysis (1:1, allowing for replacement of controls), and $35.6 \%$ of cases (9402 case-control pairs) in the third sensitivity analysis (1:2, allowing for replacement of controls). Supplementary table 9 shows the characteristics of the population in these three matching analyses. Overall, vaccine effectiveness was comparable to the findings of the main analysis, with varying precision. Vaccine effectiveness against symptomatic covid-19 was $41.6 \%$ (26.9\% to $53.3 \%)$ in the first sensitivity analysis ( $\mathrm{n}=15900), 48.6 \%$ (38.9\% to $56.8 \%$ ) in the second sensitivity analysis $(n=26566)$, and $47.8 \%$ (38.2\% to 56.0\%) in the third

\section{Case-control Vaccine effectiveness, sets $\%(95 \% \mathrm{Cl})$}

\section{Cases with symptoms}

70-74 years 7024

$75-79$ years 3519

$\geq 80$ years 2740

Cases admitted to hospital

70-74 years 3263

75-79 years 1928

$\geq 80$ years 1852

Deaths

70-74 years 1517

75-79 years 1005

$\geq 80$ years $\quad 1027$

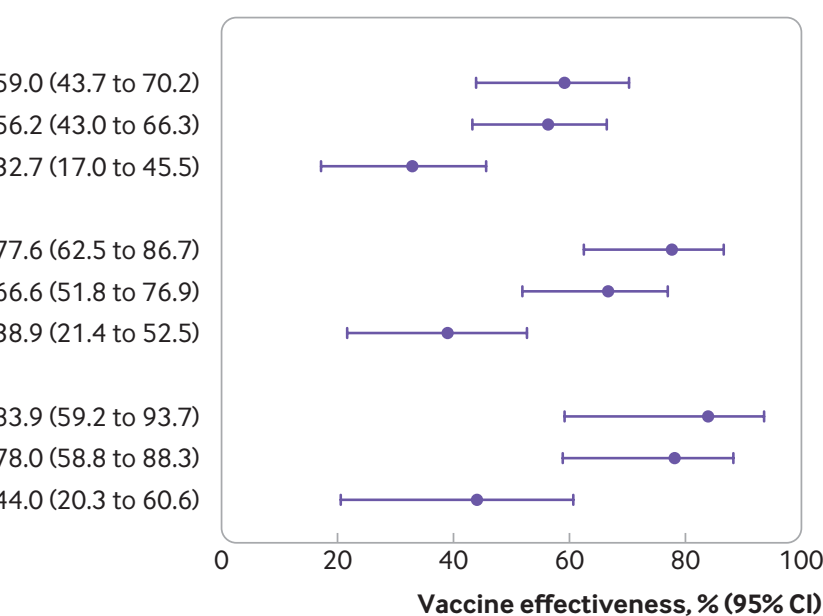

Fig 3 | Adjusted vaccine effectiveness $\geq 14$ days after the second dose of CoronaVac (Sinovac Biotech) for subgroups of adults aged $\geq 70$ years. Estimates of vaccine effectiveness were obtained from a conditional logistic regression model that included covariates of age and number of comorbidities and incorporated an interaction term between the category of interest and the period $\geq 14$ days after the second dose 
sensitivity analysis ( $n=28206$; supplementary tables 10-13). The same pattern of vaccine effectiveness observed in the main analysis when stratified by age and for severe outcomes was observed in the three sensitivity analyses (supplementary tables 10 and 14-16).

\section{Discussion}

In this test negative case-control study we found that the effectiveness of a two dose schedule of CoronaVac in the real world was $47 \%$ against symptomatic covid-19, 56\% against covid-19 associated hospital admissions, and $61 \%$ against covid-19 associated deaths among those aged $\geq 70$ years during a gamma variant associated epidemic in Brazil. Furthermore, we have addressed several evidence gaps for the use of CoronaVac: vaccination showed an effectiveness against covid-19, including associated severe outcomes, in the setting of widespread transmission of the gamma variant, which was similar to that found in the Brazilian randomised controlled trial conducted before the emergence of the gamma variant ${ }^{5}$; a single dose of CoronaVac was associated with low protection against symptomatic covid-19 or hospital admission; and vaccine effectiveness was observed to decline with increasing age among adults aged $\geq 70$ years.

\section{Research in context}

A key evidence gap, as raised in the WHO EUL for CoronaVac, ${ }^{11}$ has been the effectiveness of this vaccine in the elderly population, because this age group was not well represented in Brazilian and Turkish randomised controlled trials. ${ }^{5} 71011$ We found that two doses of CoronaVac administered at an average interval of four weeks had an overall effectiveness against symptomatic covid-19 of $47 \%$ (39\% to $54 \%$ ) in a population with a mean age of 76 years. This estimate is lower than the efficacy of $84 \%$ (95\% confidence interval $65 \%$ to $92 \%$ ) reported in the Turkish trial, with a participant median age of 45 years and two week dosing interval ${ }^{7}$; and comparable to the efficacy of $51 \%$ (95\% confidence interval 36\% to 62\%) from the Brazilian trial in healthcare workers, with a participant mean age of 39 years and two week dosing interval. ${ }^{5}$ Additionally, a cohort study in Chile reported an effectiveness for CoronaVac of $66.6 \%$ (95\% confidence interval $65.4 \%$ to $67.8 \%$ ) in those aged $\geq 60$ years. It is not clear whether the observed differences are related to the age distribution, dosing interval, risk of infection in the community, or the presence of the gamma variant of concern, which was not prevalent during the trials' follow-up periods and was responsible for only $28.6 \%$ of genotyped infections in Chile during the study period. ${ }^{1012}$

Among elderly people in our study, we observed a statistically significant decline in vaccine effectiveness against symptomatic covid-19 with increasing age, from $59.0 \%(43.7 \%$ to $70.2 \%)$ in those aged $70-74$ year to $32.7 \%(17.0 \%$ to $45.5 \%)$ in those aged $\geq 80$ years. These findings parallel real world evidence for the BNT162b2 mRNA vaccine, which showed reduced effectiveness in residents of long term care facilities in Denmark, ${ }^{41}$ skilled nursing facilities in the USA, ${ }^{42}$ and the general population aged $\geq 70$ years in Finland ${ }^{43}$ and $\geq 80$ years in Israel. ${ }^{44}$ As well as having a slower immune response and lower peak of neutralising antibodies than younger populations, elderly people seem to have faster decay of antibody titres. ${ }^{4}$ Together, these findings suggest that specific vaccines or vaccination schedules might be required to effectively vaccinate the very elderly ( $\geq 80$ years) population against covid-19.

Vaccine effectiveness was greater against severe outcomes than against symptomatic covid-19 in all age subgroups among elderly people. This finding, consistent with the findings from randomised controlled trials and observational studies for multiple covid-19 vaccines and across settings, ${ }^{5691012}$ suggests that vaccination will reduce morbidity and mortality among elderly people even if effectiveness at preventing infections is reduced. The direct comparison of the effectiveness against hospital admission with other vaccines and between countries is not straightforward, because hospital admission depends on admission triage policies, which change according to age and hospital bed availability. Therefore, someone older than 80 years with symptomatic covid-19 has a higher likelihood of being admitted compared with younger patients even if the disease is not severe, and this likelihood varies between public and private facilities and whether the health system is overwhelmed. ${ }^{13}$ Thus, we cannot generalise our findings for protection against hospital admission without considering this context. We evaluated vaccine effectiveness at the individual level, not accounting for the indirect effect and the total effect from the vaccination campaign. A preliminary aggregated analysis using weekly times series of covid-19 deaths in Brazil found a relative decrease in mortality among those aged $\geq 70$ years compared with all ages after vaccination with CoronaVac and ChAdOx1 nCov-19, ${ }^{45}$ suggesting a discernible impact of vaccination on mortality at the population level. Additional investigation is required to determine the duration of protection conferred by CoronaVac. ${ }^{7} 1923$

The absence of demonstrable effectiveness of CoronaVac until completion of the two dose regimen has profound implications for use of this vaccine in response to an epidemic. In contrast with covid-19 vaccines that confer protection after the first dose, ${ }^{9} 46$ CoronaVac showed low effectiveness until after the second dose (more than four weeks after the first dose). ${ }^{19}$ Our findings suggest that in countries where CoronaVac supplies are constrained and there is high SARS-CoV-2 transmission, vaccination should prioritise completion of the two dose regimen among the highest risk populations and avoid being expanded to broader segments of the population for whom provisions for a second dose have not been secured.

Our study did not directly address the question of whether vaccination with CoronaVac is effective against gamma variant associated covid-19 because we had 
no data on whether the analysed cases were related to the gamma variant. However, 91.0\% (5054/5551) of the discordant sets in this matched case-control study were selected from 1 March to 29 April 2021, when the gamma variant accounted for $85 \%$ of the genotyped isolates during surveillance in São Paulo state. A test negative study in Canada evaluated adults aged $\geq 70$ years and estimated an adjusted vaccine effectiveness of single dose mRNA vaccines of $61 \%$ (95\% confidence interval $45 \%$ to $72 \%$ ) against the gamma variant of concern compared with $72 \%$ (58\% to $81 \%$ ) for nonvariants of concern. ${ }^{47}$ Although further studies are required to determine the effectiveness of CoronaVac against the gamma variant and additional variants of concern, our findings provide supportive evidence for the use of CoronaVac in countries in South America that are experiencing epidemics due to extensive spread of the gamma variant ${ }^{22}$ and are using CoronaVac as part of a mass vaccination campaign in response to the epidemic.

\section{Strengths and limitations of this study}

This study has several strengths, which include the large sample size and geospatial coverage, comprising the State of São Paulo with 46 million inhabitants distributed across 645 municipalities. We implemented a prespecified publicly available protocol, which is in accordance with the recent WHO guideline for evaluation of covid-19 vaccine effectiveness. ${ }^{23}$ Using a test negative design, we have dealt with biases that affect observational studies on vaccine effectiveness, such as health seeking behaviour and access. Additionally, after matching and adjustment, the bias indicator association between recent vaccination with a single dose 0-13 days before sample collection was close to null, suggesting that the underlying risk of testing positive for SARS-CoV-2 did not differ between vaccinated and unvaccinated people. ${ }^{835} 36$ ${ }^{48}$ Finally, we performed three sensitivity analyses for the matching procedure, which yielded comparable estimates to those of the main analysis, resulting in increased precision and showing the robustness of our vaccine effectiveness estimation.

Our study had limitations. We could not assess the influence of a previous SARS-CoV-2 infection on vaccine effectiveness because passive surveillance identified too few people with a positive RT-PCR or rapid antigen test result before the study period. Before the start of the vaccination campaign, the estimated seroprevalence of covid-19 in inhabitants aged $\geq 60$ years in the capital of São Paulo state was 19.9\% (14.9\% to $29.9 \%$ ) in January 2021 . $^{49}$ Our estimates of vaccine effectiveness might therefore be subject to downward bias, as unvaccinated people were at lower risk of reinfection. We attempted to exclude false negative RTPCR test results by excluding as controls those with a subsequent positive test result within 14 days after the initial test and including only tests performed within 10 days of symptom onset. ${ }^{23}$ However, we cannot rule out some level of misclassification, although it is likely to be non-differential and thus would bias the estimate towards the null. In addition, we restricted our study population to elderly people because they were a priority group for vaccination and received the majority of CoronaVac doses during the initial stages of the vaccination campaign in Brazil; as a result, it was not possible to compare the effectiveness of CoronaVac between older and younger populations directly. Our analyses were also limited by the lack of more refined covariates, such as frailty, chronic illness status, and nursing home residence status, which could influence vaccine effectiveness in very elderly people and in itself would not be addressed by age and reported comorbidities. Finally, we cannot exclude the possibility of time varying changes in behaviour, non-drug interventions, or testing practices among participants. We tried to control for these by matching on time of RT-PCR testing ( \pm 3 days),$^{21}$ geography (ie, municipality of residence), and self-reported race, which is strongly associated with socioeconomic position in Brazil. When we tried to further adjust for unmeasured confounding by adjusting for the day of year, estimates of vaccine effectiveness remained similar.

\section{Conclusion}

This study found that a two dose schedule of CoronaVac was $47 \%$ effective in preventing symptomatic covid-19, with higher effectiveness against severe clinical outcomes, among elderly people aged $\geq 70$ years in a setting with extensive transmission of the gamma variant. The delayed onset of vaccine mediated protection, however, underscores the need to prioritise vaccine supplies and maximise the number of people who complete the two dose schedule, when CoronaVac is used as part of a mass vaccination campaign that is implemented in response to a covid-19 epidemic.

\section{AUTHOR AFFILIATIONS}

${ }^{1}$ Barcelona Institute for Global Health, ISGlobal, Barcelona, Spain

${ }^{2}$ Pulmonary Division, Heart Institute (InCor), Hospital das Clinicas

HCFMUSP, Faculdade de Medicina, Universidade de São Paulo, São Paulo, SP, Brazil

${ }^{3}$ Department of Biostatistics, College of Public Health \& Health Professions, University of Florida, Gainesville, FL, USA

${ }^{4}$ Department of Epidemiology of Microbial Diseases, Yale School of Public Health, New Haven, CT, USA

${ }^{5}$ Disease Control Coordination of the São Paulo State Department of Health, São Paulo, Brazil

${ }^{6}$ Municipal Health Secretary of Manaus, Brazil, AM, Brazil

${ }^{7}$ Pan American Health Organization, Brasília, DF, Brazil

${ }^{8}$ Universidade de Brasília, Brasília, DF, Brazil

${ }^{9}$ Department of Laboratory Medicine, Yale University School of Medicine, New Haven, CT, USA

${ }^{10}$ State University of Mato Grosso do Sul - UEMS, Dourados, MS, Brazil

${ }^{11}$ Universidade Federal de Mato Grosso do Sul - UFMS, Campo Grande, MS, Brazil

${ }^{12}$ National Institute for Science and Technology for Health Technology Assessment, Porto Alegre, RS, Brazil

${ }^{13}$ Health Secretariat of the State of São Paulo, São Paulo, Brazil

${ }^{14}$ Division of Infectious Diseases and Geographic Medicine, Stanford University, Stanford, CA, USA

${ }^{15}$ Department of Biology, University of Florida, Gainesville, FL, USA ${ }^{16}$ Emerging Pathogens Institute, University of Florida, Gainesville, FL, USA 
${ }^{17}$ Instituto Gonçalo Moniz, Fundação Oswaldo Cruz, Salvador, BA, Brazil

${ }^{18}$ Fiocruz Mato Grosso do Sul, Fundação Oswaldo Cruz, Campo Grande, MS, Brazil

We thank the Pan American Health Organization for its support and the São Paulo state for making the databases available for analysis.

Contributors: All authors conceived the study. OTR and MDTH contributed equally as first authors and JRA, DATC, AIK, and JC contributed equally as senior authors. OTR, MDTH, and MD completed analyses with guidance from JRA, DATC, AIK, and JC. MSST, OFPP, OTR, and MDTH curated and validated the data. OTR and MDTH wrote the first draft of the manuscript. TLD, RCP, OFPP, EFMV, MA, RS, ICG, and WNA provided supervision. All authors contributed to, and approved, the final manuscript. JC is the guarantor. The corresponding author attests that all listed authors meet authorship criteria and that no others meeting the criteria have been omitted.

Funding: No external funding was provided for this study. JC is supported by the Oswaldo Cruz Foundation (Edital Covid-19 resposta rápida: 48111668950485$)$. OTR is funded by a Sara Borrell fellowship (CD19/00110) from the Instituto de Salud Carlos III. OTR acknowledges support from the Spanish Ministry of Science and Innovation through the Centro de Excelencia Severo Ocho 2019-2023 programme and from the Generalitat de Catalunya through the Centres de Recerca de Catalunya (CERCA) programme. These institutions had no role in the study design, data collection, data analysis, data interpretation, or writing of the report. The health secretary of State of São Paulo and information technology bureau of the São Paulo state government reviewed the data and findings of the study, but the academic authors retained editorial control.

Competing interests: All authors have completed the ICMJE uniform disclosure form at www.icmje.org/coi disclosure.pdf and declare: no support from any organisation for the submitted work; no financial relationships with any organisations that might have an interest in the submitted work in the previous three years; no other relationships or activities that could appear to have influenced the submitted work.

Ethics approval: This study was approved by the ethical committee for research of Federal University of Mato Grosso do Sul (CAAE: 43289221.5.0000.0021).

Data sharing: Deidentified databases as well as the R codes will be deposited in the repository https://github.com/juliocroda/ VebraCOVID-19 on publication of this article.

The lead author (the manuscript's guarantor) affirms that the manuscript is an honest, accurate, and transparent account of the study being reported; that no important aspects of the study have been omitted; and that any discrepancies from the study as originally planned have been explained.

Dissemination to participants and related patient and public communities: The findings of this study will be disseminated through presentations to the São Paulo state health department and the national immunisation programme, as well as the World Health Organization. Blogs, press releases, and social media dissemination will also be prepared for public dissemination

Provenance and peer review: Not commissioned; externally peer reviewed.

This is an Open Access article distributed in accordance with the Creative Commons Attribution Non Commercial (CC BY-NC 4.0) license, which permits others to distribute, remix, adapt, build upon this work non-commercially, and license their derivative works on different terms, provided the original work is properly cited and the use is noncommercial. See: http://creativecommons.org/licenses/by-nc/4.0/.

1 Dong E, Du H, Gardner L. An interactive web-based dashboard to track COVID-19 in real time. Lancet Infect Dis 2020;20:533-4. doi:10.1016/S1473-3099(20)30120-1

2 O'Driscoll M, Ribeiro Dos Santos G, Wang L, et al. Agespecific mortality and immunity patterns of SARS-CoV-2. Nature 2021;590:140-5. doi:10.1038/s41586-020-2918-0

3 Bajaj V, Gadi N, Spihlman AP, Wu SC, Choi CH, Moulton VR. Aging, Immunity, and COVID-19: How Age Influences the Host Immune Response to Coronavirus Infections? Front Physiol 2021;11:571416. doi:10.3389/fphys.2020.571416

4 Collier DA, Ferreira IATM, Kotagiri P, et al. Age-related immune response heterogeneity to SARS-CoV-2 vaccine BNT162b2. Nature 2021; published online June 30. doi:10.1038/s41586-021-03739-1

5 Palacios R, Batista AP, Albuquerque CSN et al. Efficacy and Safety of a COVID-19 Inactivated Vaccine in Healthcare Professionals in Brazil: The PROFISCOV Study.SSRN Journal2021. doi:10.2139/ ssrn.3822780
6 Voysey M, Clemens SAC, Madhi SA, et al, Oxford COVID Vaccine Trial Group. Safety and efficacy of the ChAdOx1 nCoV-19 vaccine (AZD1222) against SARS-CoV-2: an interim analysis of four randomised controlled trials in Brazil, South Africa, and the UK. Lancet 2021;397:99-111. doi:10.1016/S0140-6736(20)32661-1

7 Tanriover MD, Doğanay HL, Akova M, et al, CoronaVac Study Group. Efficacy and safety of an inactivated whole-virion SARS-CoV-2 vaccine (CoronaVac): interim results of a double-blind, randomised, placebo-controlled, phase 3 trial in Turkey. Lancet 2021;398:21322. doi:10.1016/S0140-6736(21)01429-X

8 Lopez Bernal J, Andrews N, Gower C, et al. Effectiveness of the PfizerBioNTech and Oxford-AstraZeneca vaccines on covid-19 related symptoms, hospital admissions, and mortality in older adults in England: test negative case-control study. BMJ 2021;373:n1088. doi:10.1136/bmj.n1088

9 Dagan N, Barda N, Kepten E, et al. BNT162b2 mRNA Covid-19 Vaccine in a Nationwide Mass Vaccination Setting. N EnglJ Med 2021;384:1412-23. doi:10.1056/NEJMoa2101765

10 Strategic Advisory Group of Experts on Immunization - SAGE (WHO). Evidence Assessment: Sinovac/CoronaVac COVID-19 vaccine. Report from 29/04/2021. https://cdn.who.int/media/docs/default-source/ immunization/sage/2021/april/5 sage29apr2021 criticalevidence sinovac.pdf (accessed May 26, 2021).

11 Strategic Advisory Group of Experts on Immunization - SAGE (WHO). Interim recommendations for use of the inactivated CovID-19 vaccine, Coronavac, developed by Sinovac. 2021; published online May 24. https://apps.who.int/iris/bitstream/ handle/10665/341454/WHO-2019-nCoV-vaccines-SAGErecommendation-Sinovac-CoronaVac-2021.1-eng.pdf.

12 Jara A, Undurraga EA, González C, et al. Effectiveness of an Inactivated SARS-CoV-2 Vaccine in Chile. N Engl J Med 2021 doi:10.1056/NEJMoa2107715

13 Ranzani OT, Bastos LSL, Gelli JGM, et al. Characterisation of the first 250,000 hospital admissions for COVID-19 in Brazil: a retrospective analysis of nationwide data. Lancet Respir Med 2021;9:407-18. doi:10.1016/S2213-2600(20)30560-9

14 Lamarca AP, de Almeida LGP, Francisco R da S, et al. Genomic surveillance of SARS-CoV-2 tracks early interstate transmission of P.1 lineage and diversification within P.2 clade in Brazil.medRxiv, 2021. doi:10.1101/2021.03.21.21253418

15 Naveca FG, Nascimento V, de Souza VC, et al. COVID-19 in Amazonas, Brazil, was driven by the persistence of endemic lineages and P.1 emergence Nat Med 2021:27:1230-8 doi:10.1038/s41591-021 01378-7

16 Faria NR, Mellan TA, Whittaker C, et al. Genomics and epidemiology of the P.1 SARS-CoV-2 lineage in Manaus, Brazil. Science 2021;372:815-21. doi:10.1126/science.abh2644

17 Jangra S, Ye C, Rathnasinghe R, et al, Personalized Virology Initiative study group. SARS-CoV-2 spike E484K mutation reduces antibody neutralisation. Lancet Microbe 2021:2:e283-4 doi:10.1016/S26665247(21)00068-9

18 Hoffmann M, Arora P, Groß R, et al. SARS-CoV-2 variants B.1.351 and P.1 escape from neutralizing antibodies. Cell 2021:184:2384-2393. e12. doi:10.1016/j.cell.2021.03.036

19 Souza WM, Amorim MR, Sesti-Costa R, et al. Neutralisation of SARSCoV-2 lineage P.1 by antibodies elicited through natural SARS-CoV-2 infection or vaccination with an inactivated SARS-CoV-2 vaccine: an immunological study. Lancet Microbe 2021;•••. doi:10.1016/ S2666-5247(21)00129-4

20 Freitas ARR, Beckedorff OA, Cavalcanti LP de G, et al. The emergence of novel SARS-CoV-2 variant P.1 in Amazonas (Brazil) was temporally associated with a change in the age and sex profile of COVID-19 mortality: A population based ecological study. The Lancet Regional Health - Americas 2021. doi:10.1016/j.lana.2021.100021

21 Bastos LS, Ranzani OT, Souza TML, Hamacher S, Bozza FA COVID-19 hospital admissions: Brazil's first and second waves compared. Lancet Respir Med 2021;9:e82-3. doi:10.1016/S22132600(21)00287-3

22 GISAID - hCov19 Variants. https://www.gisaid.org/hcov19-variants/ (accessed June 20, 2021)

23 Patel MK, Bergeri I, Bresee JS, et al. Evaluation of post-introduction COVID-19 vaccine effectiveness: Summary of interim guidance of the World Health Organization. Vaccine 2021:39:4013-24. doi:10.1016/j.vaccine.2021.05.099

24 Freire FHM de A, Gonzaga MR. Gomes MMF. Projeções populacionais por sexo e idade para pequenas áreas no Brasil. RELAP 2019;14:124-49. doi:10.31406/relap2020.v14.i1.n26.6

25 SEADE Foundation - São Paulo State. CORONAVIRUS - CASOS EM SP. Fundação SEADE. https://www.seade.gov.br/coronavirus/ (accessed May 9, 2021).

26 Brazilian Ministry of Health. Campanha Nacional de Vacinação contra a Covid-19. Décimo oitavo informe técnico. https:// www.conasems.org.br/wp-content/uploads/2021/04/20adistribuic\%CC\%A7a\%CC\%830_Campanha_Nacional_de_Vacinacao contra_a_Covid_19.pdf (accessed May 25, 2021). 
27 De Serres G, Skowronski DM, Wu XW, Ambrose CS. The test-negative design: validity, accuracy and precision of vaccine efficacy estimates compared to the gold standard of randomised placebo-controlled clinical trials. Euro Surveill 2013;18:20585. doi:10.2807/15607917.ES2013.18.37.20585

28 Schwartz LM, Halloran ME, Rowhani-Rahbar A, Neuzil KM, Victo $J C$. Rotavirus vaccine effectiveness in low-income settings: An evaluation of the test-negative design. Vaccine 2017;35:184-90. doi:10.1016/j.vaccine.2016.10.077

29 Jackson ML, Chung JR, Jackson LA, et al. Influenza Vaccine Effectiveness in the United States during the 2015-2016 Season. N Engl / Med 2017:377:534-43. doi:10.1056/NEJMoa1700153

30 Lopez Bernal J, Andrews N, Gower C, et al. Effectiveness of Covid-19 Vaccines against the B.1.617.2 (Delta) Variant. N EnglJ Med 2021;385:585-94. doi:10.1056/NEJMoa2108891

31 Brazilian Ministry of Health. Epidemiologic Surveillance Guide. National Emergency of Public Health Concern due to the COVID-19 disease. https://www.conasems.org.br/ wp-content/uploads/2021/03/Guia-de-vigila\%CC\%82nciaepidemiolo\%CC\%81gica-da-covid_19_15.03_2021.pdf (accessed May 25, 2021)

32 Brazilian Institute of Geography and Statistics - IBGE. Ethnic and race characteristics of the population. Classifications and identities. 2013. https://biblioteca.ibge.gov.br/visualizacao/livros/liv63405.pdf (accessed May 24, 2021).

33 Bueno SM, Abarca K, González PA, et al. Interim report: Safety and immunogenicity of an inactivated vaccine against SARS-CoV-2 in healthy chilean adults in a phase 3 clinical trial.medRxiv, 2021. doi:10.1101/2021.03.31.21254494

$34 \mathrm{Wu}$ Z, Hu Y, Xu M, et al. Safety, tolerability, and immunogenicity of an inactivated SARS-CoV-2 vaccine (CoronaVac) in healthy adults aged 60 years and older: a randomised, double-blind, placebo-controlled, phase 1/2 clinical trial. Lancet Infect Dis 2021;21:803-12. doi:10.1016/S1473-3099(20)30987-7

35 Hitchings MDT, Lewnard JA, Dean NE, et al. Use of recently vaccinated individuals to detect bias in test-negative case-control studies of COVID-19 vaccine effectiveness.medRxiv , 2021 doi:10.1101/2021.06.23.21259415

36 Lewnard JA, Patel MM, Jewell NP, et al. Theoretical Framework for Retrospective Studies of the Effectiveness of SARS-CoV-2 Vaccines. Epidemiology 2021;32:508-17. doi:10.1097/ EDE.0000000000001366

37 Sullivan SG, Tchetgen Tchetgen EJ, Cowling BJ. Theoretical Basis of the Test-Negative Study Design for Assessment of Influenza Vaccine Effectiveness. Am J Epidemiol 2016;184:345-53. doi:10.1093/aje/ kww064

38 Pearce N. Analysis of matched case-control studies. BMJ 2016;352:i969. doi:10.1136/bmj.i969

39 Andrejko KL, Pry J, Myers JF, et al, California COVID-19 Case-Control Study Team. Prevention of COVID-19 by mRNA-based vaccines within the general population of California. Clin Infect Dis 2021. doi:10.1093/cid/ciab640
40 Crowe E, Pandeya N, Brotherton JML, et al. Effectiveness of quadrivalent human papillomavirus vaccine for the prevention of cervical abnormalities: case-control study nested within a population based screening programme in Australia. BMJ 2014;348:g1458. doi:10.1136/bmj.g1458

41 Moustsen-Helms IR, Emborg H-D, Nielsen J, et al. Vaccine effectiveness after 1 st and 2 nd dose of the BNT162b2 mRNA Covid-19 Vaccine in long-term care facility residents and healthcare workers - a Danish cohort study.medRxiv, 2021. doi.10.1101/2021.03.08.21252200

42 Britton A, Jacobs Slifka KM, Edens C, et al. Effectiveness of the PfizerBioNTech COVID-19 Vaccine Among Residents of Two Skilled Nursing Facilities Experiencing COVID-19 Outbreaks - Connecticut, Decembe 2020-February 2021. MMWR Morb Mortal Wkly Rep 2021;70:396401. doi:10.15585/mmwr.mm7011e3

43 Baum U, Poukka E, Palmu AA, Salo H, Lehtonen TO, Leino T. Effectiveness of vaccination against SARS-CoV-2 infection and Covid-19 hospitalization among Finnish elderly and chronically ill - An interim analysis of a nationwide cohort study.medRxiv , 2021. doi:10.1101/2021.06.21.21258686

44 Yelin I, Katz R, Herzel E, et al. Associations of the BNT162b2 COVID-19 vaccine effectiveness with patient age and comorbidities. medRxiv, 2021 doi:10.1101/2021.03.16.21253686

45 Victora PC, Castro PMC, Gurzenda S, Medeiros AC, França GVA, Barros PAJD. Estimating the early impact of vaccination against COVID-19 on deaths among elderly people in Brazil: Analyses of routinely-collected data on vaccine coverage and mortality. EClinicalMedicine 2021;36:101036. doi:10.1016/j. eclinm.2021.101036

46 Voysey M, Costa Clemens SA, Madhi SA, et al, Oxford COVID Vaccine Trial Group. Single-dose administration and the influence of the timing of the booster dose on immunogenicity and efficacy of ChAdOx1 nCoV-19 (AZD1222) vaccine: a pooled analysis of fou randomised trials. Lancet 2021;397:881-91. doi:10.1016/S01406736(21)00432-3

47 Skowronski DM, Setayeshgar S, Zou M, et al. Single-dose mRNA vaccine effectiveness against SARS-CoV-2, including Alpha and Gamma variants: a test-negative design in adults 70 years and older in British Columbia, Canada. Clin Infect Dis 2021;ciab616. doi:10.1093/cid/ciab616

48 Hitchings MDT, Ranzani OT, Torres MSS, et al. Effectiveness of CoronaVac among healthcare workers in the setting of high SARSCoV-2 Gamma variant transmission in Manaus, Brazil: A test-negative case-control study. The Lancet Regional Health - Americas 2021; 100025. doi:10.1016/j.lana.2021.100025

49 SoroEpi Group. SoroEpi MSP study in São Paulo Capital - Phase 5 results. https://www.monitoramentocovid19.org (accessed May 17, 2021).

Supplementary information: additional tables 1-16 and figures 1-4 